\title{
Effects of Epidural Needle Rotation and Different Speeds of Injection on the Distribution of Epidural Block
}

\author{
Ozlem Sen ${ }^{1}$, N Ferah Donmez ${ }^{1}$, Dilsen Ornek ${ }^{1}$, Dilek Kalayci ${ }^{1}$, Mahmut Arslan ${ }^{1}$, Bayazit Dikmen ${ }^{1}$
}

Summary: Sen O, Donmez NF, Ornek D, Kalayci D, Arslan M, Dikmen B - Effects of Epidural Needle Rotation and Different Speeds of Injection on the Distribution of Epidural Block.

Background and objectives: This prospective, randomised study examined the effect of injection speeds for unilateral epidural anesthesia on block characteristics, hemodynamic parameters, and discharge criteria in 60 patients. Levobupivacaine $5 \%$ was administered to Group $\mathrm{F}$ over 1 min (fast) and to Group S over 3 min (slow) ( $n=30$ each) with the needle angulated at $5^{\circ}-10^{\circ}$ from the midline. Unilateral epidural block was significantly more successful in Group S than in Group F $(70.3 \%$ vs. $16 \% ; p<0.001)$. On the non-operated sides in group S, the maximal sensorial block time was shorter and the regression time for 2 segments was longer $(p<0.05)$. And the walk-out time was longer in group $F(p<0.05)$. We consider that the slow administration of local anesthetic in unilateral epidural anesthesia is more effective than rapid administration.

Keywords: Anesthesia, Epidural; Bupivacaine/levobupivacaine; Catheters, Indwelling; Injections, Epidural; Orthopedic Procedures.

(2012 Elsevier Editora Ltda. All rights reserved.

\section{INTRODUCTION}

The distribution of an epidural block cannot be controlled by gravity or patient position. Nonetheless, obtaining a preferential distribution of the epidural block towards the operative side is useful, especially when large doses of analgesics are required postoperatively to tolerate aggressive physiotherapy ${ }^{1-7}$. By evaluating the epidural catheter tip position and distribution of the injected solution by computed tomography (CT), Hogan ${ }^{1-7}$ clearly demonstrated that most epidural catheter tips are placed in an anterior or lateral position. This results in great variability in the distribution of the local anesthetic solution. Introducing the epidural needle at an angle from the midline and rotating it towards the operative side has been proposed to direct the epidural catheter towards the operative side ${ }^{6}$, and various reports have supported the clinical efficacy of such an intentional 'unilateral epidural block' 6-10.

Borghi et al. ${ }^{5}$ conducted a prospective, randomised, double-blind study to evaluate the effects of turning the Tuohy needle at $45^{\circ}$ to the operative side before threading the catheter through the needle on the distribution of the epidural

Received from Anaesthesia and Reanimation Department, Ankara Numune Training and Research Hospital, Samanpazarı, Ankara, Turkey.

1. Anaesthesia and Reanimation Department, Ankara Numune Training and Research Hospital, Ankara, Turkey

Submitted on January 16, 2012

Approved on February 23, 2012.

Correspondence to:

Dilsen Ornek, MD

Anaesthesia and Reanimation Department

Ankara Numune Training and Research Hospital

Ulku Mahallesi Talatpasa Bulvari No: 5 ,

Altindag, Ankara 06100, Turkey

E-mail: dilsenpinar@yahoo.com block ${ }^{5}$. They concluded that such rotation provided a preferential distribution of the sensory and motor block towards the operative side, reducing the volume of local anesthetic solution required to maintain postoperative analgesia.

In this randomised, controlled study, we evaluated the applicability of intentional unilateral epidural anesthesia along with the effects of the rate of administration of the local anesthetic on the unilaterality, hemodynamic parameters, and discharge time in 60 patients.

\section{MATERIALS AND METHODS}

Approval from the hospital's ethics committee and written informed consent from the patients was obtained prior to the commencement of the study. We evaluated 60 patients with ASA physical status I-III receiving epidural anesthesia for elective meniscopathy in this study. Patients with contraindications to central blocks, previous back surgery, diabetes, or severe cardiovascular and/or respiratory diseases were excluded.

Standard monitoring was used throughout the procedure, including electrocardiogram (lead II), heart rate (HR), automated non-invasive arterial blood pressure (ABP), and pulse oximetry $\left(\mathrm{SpO}_{2}\right)$ (Datex Ohmeda ADUS/5, Helsinki, Finland). All patients received $7 \mathrm{~mL} . \mathrm{kg}^{-1}$ of $0.9 \% \mathrm{NaCl}$ via a peripheral vein.

Patients were placed in the lateral decubitus position so that the limb to be operated upon was lowermost. After local infiltration with $2 \%$ lidocaine, the epidural space was located with an 18-gauge Tuohy needle (Epifix 1890; Egemen 18G/20G, zmir, Turkey) at the L3-L4 interspace using a midline approach and the saline 'loss of resistance' technique. We aimed to place the tip of the epidural needle towards the side where the block 
was required at an angle of $5^{\circ}-10^{\circ}$ from the midline. In all patients, the catheter was introduced $3-4 \mathrm{~cm}$ beyond the tip of the Tuohy needle. After negative aspiration, $3 \mathrm{~mL}$ of $2 \%$ lidocaine was injected through the catheter as a test dose. The needle was then removed, and the catheter was secured to the skin. Then, using a computer-generated sequence of numbers, patients were randomly allocated into two groups as follows: $5 \%$

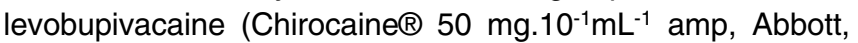
Espoo, Finland) at a volume of $10 \mathrm{~mL}$ was administered via the catheter at a fast rate (Group $\mathrm{F}, \mathrm{n}=30$ ) over 1 min or a slow rate (Group $S, n=30$ ) over 3 min. Patients in both groups were kept in a lateral decubitus position for $10 \mathrm{~min}$, following which they were placed in the supine position.

A blinded independent observer recorded the evolution of sensory and motor blocks on both sides every 5 min until the patient was deemed ready for surgery. The sensory block was assessed using the loss of pinprick sensation, whereas the motor block was assessed using a modified Bromage score ( 0 = no motor block; 1 = hip blocked; 2 = hip and knee blocked; $3=$ hip, knee, and ankle blocked). Readiness for surgery was defined as complete loss of pinprick sensation up to T10 with a modified Bromage scale $\geq 2$ on the surgical side. After readiness for surgery was achieved, the evolution of sensory and motor blocks was evaluated every 15 min until 2-segment regression of the sensory level was noted.

Tourniquet implementation was permitted when sensorial block reached the $T_{12}$ segment on the region to be operated. The tourniquet implementation time was recorded as the time at which the operation was started (TSO).

Fentanyl was administered to patients experiencing perioperative pain. If the pain persisted, $20-50 \mathrm{mg}$ of propofol infusion was administered. The doses of fentanyl and propofol were carefully recorded.

The hemodynamic parameters and oxygen saturation $\left(\mathrm{SpO}_{2}\right)$ was recorded at $5 \mathrm{~min}$ intervals during the first $30 \mathrm{~min}$ of the operation; subsequently, they were recorded at $15 \mathrm{~min}$ intervals. A decrease in the systolic arterial blood pressure $\geq 30 \%$ from the baseline was considered as clinically relevant hypotension and was treated with intravenous (IV) crystalloid infusion. If volume expansion was not effective, 2-5 mg IV phenylephrine was administered. A heart beat rate below 50 beats per min was regarded as bradycardia and treated with $0.5 \mathrm{mg}$ of atropine. Hypotension and bradycardia were recorded as side effects of the anesthesia.

The degree of pain, the need for rescue analgesia, the distribution of the sensory and motor blocks on the operative and non-operative sides, and the occurrence of any undesired side effects were recorded at 1, 2, 4, 6, 12 and $24 \mathrm{~h}$ after surgery. The degree of pain was assessed using a visual analogue scale (VAS). Intramuscular (IM) diclofenac (75 mg) was administered to patients with a pain score of VAS $>4$. The occurrence of bladder globus with urinary retention requiring bladder catheterization and the walk-out criteria (WOC) (raising the foot without any help and ability to perform deep knee stretching exercise) were recorded. Mental vigilance, stable vital findings, absence of nausea and vomiting, controllable pain, fulfilment of walk-out criteria, and appropriate urination time were established as the discharge criteria. To calculate the required sample size, we considered the results of a previous study ${ }^{10}$. We hoped to detect a 3-dermatome difference in the maximum sensory level between the operative and non-operative sides in the 2 groups; according to the 0.75 effect size for the standard deviation ratio calculated from the data of the pilot study, 24 patients per group were required for detecting this difference with a 2 -tailed $\alpha$-error of $5 \%$ and a $\beta$-error of $20 \%$.

Statistical analysis was performed using the statistical software package Systat 11.5 (SPSS Inc., Chicago, IL). The normal distribution of the considered variables was first evaluated using the Kolmogorov-Smirnov test. Data were presented as mean $( \pm S D$ ) or median (range), or patient number (\%). Statistically significant differences between the 2 groups were analysed using Student's t-test or Mann Whitney U test. Statistically significant differences between recurrent measurements conducted in the 2 groups were assessed using a repeated measures analysis of variance or Friedman test; significant results were analysed using Bonferroni's correction for determining the time of measurement causing the difference. Results related to all intra-group comparisons were presented after applying Bonferroni's correction. For categorical comparisons, the chi-square and Fisher's definitive tests were used. Ordinal data are presented as percentages. A p-value $\leq$ $5 \%$ was considered as statistically significant.

\section{RESULTS}

Two patients were excluded from the analysis since they suffered a dural puncture, and spinal anesthesia was subsequently administered. No difference in age, weight, height, duration of surgery, gender, or ASA physical status distribution was observed between the 2 groups (Table I).

Table I - Patient Demographics and Duration of Surgery

\begin{tabular}{llll}
\hline & $\begin{array}{l}\text { Group F } \\
(\mathrm{n}=30)\end{array}$ & $\begin{array}{l}\text { Group S } \\
(\mathrm{n}=30)\end{array}$ & $\mathrm{p}$ \\
\hline Age (years) & $\begin{array}{l}42.9 \pm 11.88 \\
(18-65)\end{array}$ & $\begin{array}{l}44.5 \pm 10.47 \\
(26-64)\end{array}$ & 0.567 \\
Weight (kg) & $80.36 \pm 10.35$ & $78.40 \pm 9.93$ & 0.456 \\
Height (cm) & $168.46 \pm 8.42$ & $167.36 \pm 9.23$ & 0.632 \\
Gender (M/F) & $12 / 18$ & $14 / 16$ & 0.602 \\
ASA (I/II) & $12 / 18$ & $13 / 17$ & 0.793 \\
$\begin{array}{l}\text { Operation Onset } \\
\text { Time (min) }\end{array}$ & $18.20 \pm 3.23$ & $18.23 \pm 2.60$ & 0.941 \\
$\begin{array}{l}\text { Operation Time } \\
\text { (min) }\end{array}$ & $33(17-64)$ & $40(20-76)$ & 0.088 \\
$\begin{array}{l}\text { Re-operation } \\
\text { Frequency }\end{array}$ & $1(1-3)$ & $1(1-3)$ & 0.914 \\
\hline
\end{tabular}


Unilateral epidural block was successfully obtained in five cases in Group F (16\%) and in 22 cases in Group S (73.3\%); thus, the success rate was significantly higher in the slow group $(p<0.001)$.

Figure 1 shows the evolution of the sensory levels on both the operative and non-operative sides in the two groups. For the sensory level on the operative side, no statistically significant difference was found for the time taken for sensorial block onset, maximum sensorial block level, 2-segment regression, for the maximum sensorial block level reached and the dermatome numbers between the two groups $(p>0.05)$. For the non-operative side, the sensorial block onset time and maximum sensorial block level were also similar in both the groups; however, in Group S, the 2-segment regression time was longer and the dermatome number against time was lesser (Figure 2) as compared to that in Group F.

In the intra-group comparisons of Group $F$, the sensorial block onset time was shorter $(p=0.001)$ and the number of dermatomes maintained under sensorial block was higher $(p=0.000)$ on the operative side. However, the regression time $(p=0.568)$ and maximum sensorial block level $(p=0.162)$ were similar for both sides.

In the intra-group comparisons of Group S, the sensorial block onset time was shorter $(p=0.017)$, while the dermatome number maintained under sensorial block $(p<0.001)$ (Figure 3 ) and the maximum sensorial block level $(p=0.002)$ were higher on the operative sides. However, the regression time $(p=0.063)$ was similar for both sides.

Figure 3 shows the distribution of the motor blockade on both the operative and non-operative sides; no statistically significant difference was found between the two groups for the motor blockade characteristics. In the intra-group comparisons of Group F, the maximum motor block level was higher $(p<0.001)$ on the operative sides; however, the motor block onset time ( $p=0.233$ ) was similar for both sides. Similarly, in the intra-group comparisons of Group $S$, the maximum motor block level ( $p<0.001)$ was higher on the operative sides; however, the motor block onset time $(p=0.109)$ was similar for both sides.

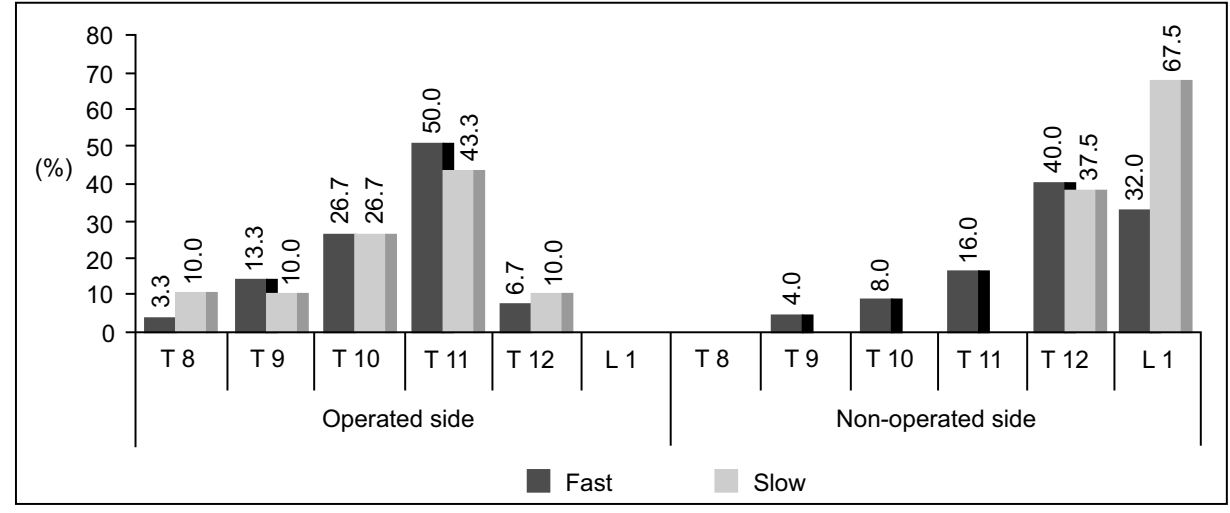

Figure 1 - Distribution of Sensorial Block Levels on Operated Side or Non-Operated Side Between Groups (Fast: Group F; Slow: Group S).

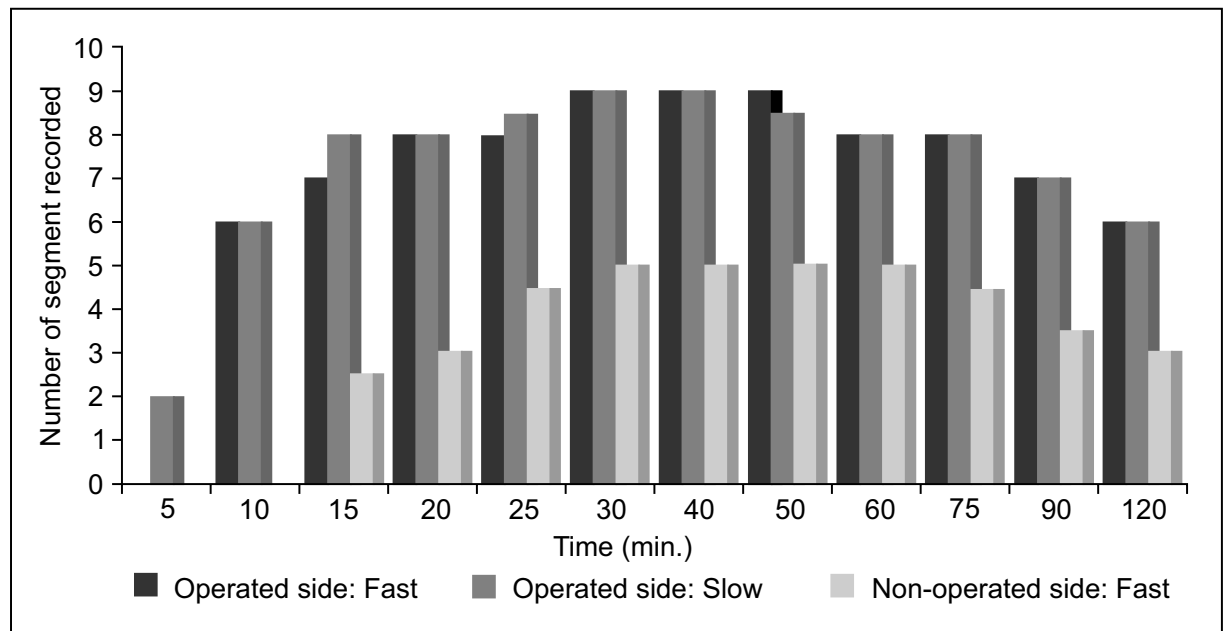

Figure 2 - Number of Segments Recorded in Time. 
There were no differences between the two groups for the heart rate and mean blood pressure changes at different measurement times (Figures 4 and 5). No patient required IV administration of vasoconstrictive drugs, and hypotension was successfully treated with volume expansion. No severe side effects were reported in either group, and the incidence of side effects was similar in the two groups $(p=0.739)$. In

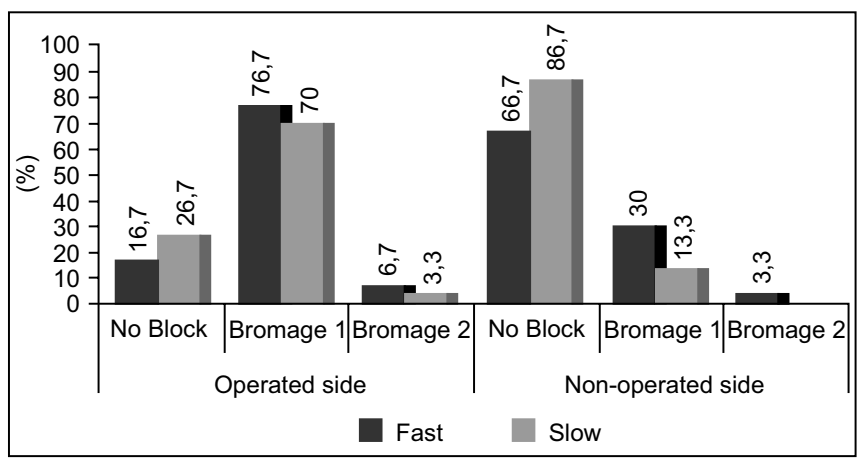

Figure 3 - Distribution of Motor Block Levels on Operated Side or Non-Operated Side Between Groups (Fast: Group F; Slow: Group S).

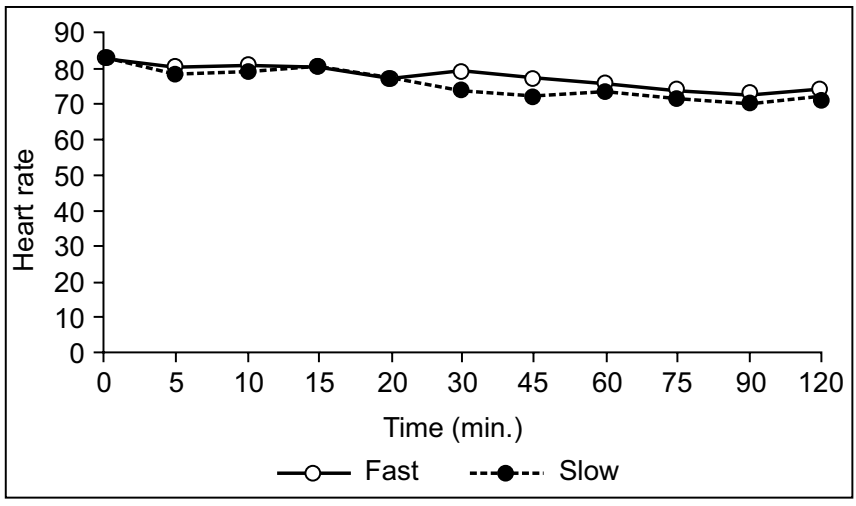

Figure 4 - Distribution of Heart Rate (Fast: Group F; Slow: Group S).

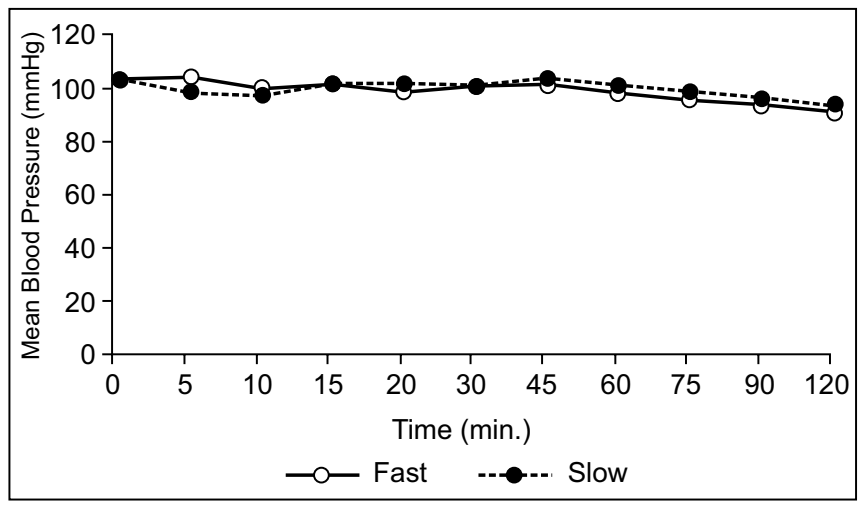

Figure 5 - Distribution of Mean Blood Pressures (Fast: Group F; Slow: Group S). the postoperative period, urinary retention requiring bladder catheterization was observed in two patients of Group $F$ and in one patient of Group S, one patient each in Group $F$ and $S$ experienced bradycardia; two patients in each group had nausea; and one in each group had vasovagal reflex.

No differences in the quality of pain, primary analgesic requirement time, and urination time were observed between the two groups; however, the walk-out time in Group S was longer (Table II). While age and urination time were not correlated $(r=-0.077$ and $p=0.553)$, gender and urination time were observed to be correlated, i.e. the urination time in women (335 (100-707) $\mathrm{min})$ was shorter than that in men (393 $(140-780) \mathrm{min})(p=0.015)$. The walk-out time was similar for both genders $(p=0.627)$.

Table II - Post-operative Evaluation (mean \pm SD)

\begin{tabular}{llll}
\hline & Group F $(\mathbf{n}=\mathbf{3 0})$ & Group S $(\mathbf{n}=\mathbf{3 0})$ & $\mathbf{p}$ \\
\hline PART (min) & $398 \pm 180.61$ & $372 \pm 167.5$ & 0.679 \\
UT (min) & $368 \pm 128$ & $394 \pm 159$ & 0.853 \\
WOT (min) & $366 \pm 188$ & $279 \pm 173$ & $0.019^{\star}$ \\
\hline
\end{tabular}

${ }^{*} p<0.05$; PART: Primary analgesic requirement time; UT: Urination time; WOT: Walk-out time.

\section{DISCUSSION}

In the present study, in $75 \%$ of the patients the knee arthroscopy could be completed following the intentional unilateral epidural anesthesia without any additional anesthesia. Administration of the local anaesthetic at a slow rate (Group S) successfully achieved epidural blockade in $73.3 \%$ patients. Moreover, the block on the non-operative side was limited in the patients from Group S.

It is possible to obtain early discharge and lesser side effects with the use of local anesthetics by appropriate titration during epidural anesthesia, which also provides the advantage of lengthening the effect of anesthetics. In our study, it was used levobupivacaine, which is similar to bupivacaine in terms of anesthetic effects, but with a lower probability of cardiovascular side effects and a lower incidence of temporary sequelae as compared to lidocaine.

For single-dose administration via epidural injection, the local anesthetic solution is generally injected into the posterior area, diffusing circumferentially towards minimal tissue resistance in the cranio-caudal axis, interior-lateral axis, and around the dura ${ }^{11-13}$. The diffusion is first longitudinal, then lateral, and finally circumferential. Circumferential diffusion is the key to the development of the sensorial block.

Epidural catheters, which are used to titrate local anesthesia administration, for additional anesthetic delivery in long surgeries, and to administer post-operative analgesics, are generally not placed in the midline but rather in the lateral or 
anterolateral area ${ }^{10}$. In $5-21 \%$ of patients subjected to epidural blockade, the block has been observed to develop only unilaterally ${ }^{14,15}$. The existence of the plica mediana dorsalis ${ }^{16}$, acquired midline adhesion ${ }^{17}$, and implantation of a lateral, anterolateral or paravertebral catheter ${ }^{11,13}$, in addition to the administration of low volumes of local anaesthetics at a slow rate in the lateral decubitus position, are considered to lead to unilateral epidural block development.

Unilateral epidural blockade can also be achieved by intentionally directing the epidural catheter laterally ${ }^{3,4}$. This method affects hemodynamic parameters to a lesser extent compared to the standard epidural while achieving the necessary anesthesia or analgesia ${ }^{3}$.

Buchheit et al. ${ }^{4}$ noted that unilateral placement and blockade could be achieved by fixing the epidural pinpoint at $5^{\circ}-10^{\circ}$ of deviation towards the lateral position and by moving the catheter forward by turning the tip of the epidural needle in the direction of the limb to be anaesthetised ${ }^{4}$. Borghi et al. were able to place the catheter appropriately by turning the needle's extremity $45^{\circ}$ towards the limb to be operated after advancing the tip of the epidural needle in the midline; this method revealed significant differences in terms of the motor and sensorial blocks between the operative and non-operative sides ${ }^{5}$. Dikmen et al. were able to place the epidural catheter for post-operative analgesic purposes as described by Buchheit et al. ${ }^{4}$, and demonstrated that the total morphine consumption and urinary retention were lower when using unilateral epidural block 6 .

Epidural anesthesia is a technically more difficult procedure than spinal anesthesia, and may result in slower anesthesia, with the risk of intravascular or intrathecal injection and insufficient blockade. While recovery is faster in epidural anesthesia, the incidence of headache and patient satisfaction is similar to that in spinal anesthesia. In the present study, we carefully rotated the needle and the catheter was placed after fixing the needle tip laterally at $\stackrel{-}{-} 10^{\circ}$ in the midline to ensure that the needle was directed toward the operative limb. Using this method, we faced no technical difficulty in most cases in finding the epidural space and placing the catheter.
The anesthesia onset time was observed to be $~ 8$ min on the operative sides, which was comparable to that in spinal anesthesia ${ }^{5}$. The operation starting time in both groups was noted to be $\sim 18 \mathrm{~min}$, similar to that reported by Borghi et al. ${ }^{5}$. The maximum sensorial block level achieved in our study was, however, lower than that reported by Borghi et al. ${ }^{5}$, while the 2 -segment regression time was similar. The motor block level reached the Bromage 1 level on the operative side, while it was maintained at Bromage 0 level on the non-operative side. The findings of the present study thus demonstrate that the method used herein for catheter placement was successful in developing a unilateral epidural block.

Insufficient pain treatment negatively affects the postoperative discharge. In orthopaedic surgery, post-operative pain is known to be higher than in other surgeries ${ }^{18}$. In our study, the primary analgesic requirement time was similar in both groups, being approximately $6 \mathrm{~h}$, demonstrating that the anesthesia method applied here provided efficacious postoperative analgesia.

Urinary retention is an important factor that delays postoperative discharge. Risk factors for urinary retention include a history of post-operative urinary retention, spinal/epidural anesthesia, pelvic or urologic operation, and peri-operative catheterization ${ }^{18}$. In our study, while urination time did not differ significantly between the groups, it was shorter in women as compared to men. Urination time may vary depending on the dose of the local anesthetic used in spinal anesthesia. In spinal anesthesia with $15 \mathrm{mg}$ of bupivacaine, urination time has been reported to be as long as $428 \min ^{18}$. Our results, however, revealed a much shorter urination time.

Walking without the need of assistance is one of the safety criteria for patient discharge. Thus, in our study, we used the walk-out criteria specified by Vagadhia et al. ${ }^{19}$. The walk-out time was shorter in the group with successful unilateral epidural anesthesia.

Based on our results, we consider that the administration of a local anaesthetic at a slow rate is effective in achieving unilateral epidural block and that intentional unilateral epidural anesthesia can be successfully used for lower extremity surgery as an alternative to other anesthesia methods. 


\section{REFERENCES}

1. Pollock JE, Mulroy MF, Bent E, Polissar NL - A comparison of two regional anesthetic techniques for outpatient knee arthroscopy. Anesth Analg, 2003;97:397-401.

2. Mulroy MF, Larkin KL, Hodgson PS et al. - A comparison of spinal, epidural and general anesthesia for outpatient knee arthroscopy. Anesth Analg, 2000;91:860-864.

3. Hogan $Q-$ Epidural catheter tip position and distribution of injectate evaluated by computed tomography. Anesthesiology, 1999;90(4):964970.

4. Buchheit T, Crews JC - Lateral cervical epidural catheter placement for continuous unilateral upper extremity analgesia and sympathetic block. Reg Anesth Pain Med, 2000;25:313-317.

5. Borghi B, Agnoletti V, Ricci A et al. - A prospective, randomized evaluation of the effects of epidural needle rotation on the distribution of epidural block. Anesth Analg, 2004;98:1473-1478.

6. Dikmen B, Peker A, Horasanlı E et al. - Is intentional unilateral epidural catheterization reliable on pain management? Pain Clinic, 2005;17(4):377-382.

7. Urban MK - Anaesthesia for orthopedic surgery. Em: Miller RD, Miller's Anesthesia. Philadelphia, Churchill Livingstone, 2010:22412259.

8. White PF, Matthew RE - Ambulatory (outpatient) anesthesia: regional anesthesia for ambulatory surgery. Em: Miller RD, Miller's Anesthesia. Philadelphia, Churchill Livingstone, 2010:2419-2459.

9. Vloka JD, Hadzic A, Mulcare R et al. - Femoral and genitofemoral nerve blocks versus spinal anesthesia for outpatients undergoing long saphenous vein stripping surgery. Anesth Analg, 1997;84:749.

10. Hogan $Q$ - Epidural catheter tip position and distribution of injectate evaluated by computed tomography. Anesthesiology, 1999;90(4):964970.

11. Fumio A, Fumio $G$ - Radiographic findings of unilateral epidural block. Anesth Analg, 1996;83:519-522.

12. Boezaart AP - Computerized axial tomo-epidurographic documentation of unilateral epidural analgesia. Can J Anesth, 1989;36:697-700.
13. Usubiaga JE, Reis A, Usubiaga LE - Epidural misplacement of catheters and mechanisms of unilateral blockade. Anesthesiology, 1977;32:150-161.

14. McCrae AF, Whitfield A, McClure JH - Repeated unilateral epidural blockade. Anesthesia, 1992;47:859-861.

15. Withington DE, Weeks SK - Repeat epidural analgesia and unilateral block. Can J Anesth, 1994;41(7):568-571.

16. Gallart L, Blanco D, Samsó E, Vidal F-Clinical and radiologic evidence of the epidural plica mediana dorsalis. Anesth Analg, 1990;71:698701.

17. Fumio A, Naomi H, Masao O, Ikuo I, Ichiro N, Yasuto K, Tadahide $\mathrm{T}$ - A median epidural septum is not a common cause of unilateral epidural blockade. Anesth Analg, 1990;71:427-429.

18. Marshall SI, Chung F - Discharge criteria and complications of after ambulatory surgery. Anesth Analg, 1999;88:508-517.

19. Vaghadia $H$, Viskari $D$, Berrill $A$ - Selective spinal anesthesia for outpatient laparoscopy. Can J Anesth, 2001;48(3):256-260. 